\title{
The Change of Aquaporin 3 Expression in Colonic Tissues of Different Degrees Diarrhea Model Rats
}

\author{
Yiwei Tang ${ }^{1, a}$, Wei Zhou ${ }^{1, b}$, Jia Chen ${ }^{1, c}$, Long Xiang ${ }^{1, d^{*}}$ \\ ${ }^{1}$ Chengdu First People's Hospital, Chengdu, 610041, China \\ aemail, ${ }^{b}$ email, ${ }^{\mathrm{c} e m a i l,}$ 'email \\ ${ }^{*}$ Corresponding Author: Long Xiang
}

Keywords: Different degree of diarrhea, Colon. Aquaporin 3

\begin{abstract}
Objective: To study the change of AQP3 expression in colonic tissues of different degrees' diarrhea model rats. Methods: SD rats were randomly divided into four groups: control group, mild diarrhea group, moderate diarrhea group and severe diarrhea group. the rat models of diarrhea were established by using different concentrations of sennan intragastric administration for $7 \mathrm{~d}$; The water content of feces was measured. Using optical microscopy to observe the pathological and morphological changes in colonic tissues of different degrees' diarrhea model rats; The expression of AQP3 protein in colonic tissues were detected by immunohistochemistry assay and Western blot. The expression of AQP3 mRNA were detected by RT-PCR. Results The water contents of feces were significantly higher in diarrhea model groups $(\mathrm{P}<0.01)$, pairwise comparison, the difference were statistically significant $(\mathrm{P}<0.05)$; HE staining showed, compared with the control group, there were more sticky secretions in diarrhea groups, glandular cells of mucous membrane laye markedly swollen, we could see a large number of congested capillaries in submucosa, the heavier the degree of diarrhea, the more obvious change; Immunohistochemistry assay and Western blot, RT-PCR results showed that compared with the control group, AQP3 protein and AQP3 mRNA expression in colon were reduced in diarrhea groups $(\mathrm{P}<0.01)$, and the heavier the degree of diarrhea, the lower expression quantity $(\mathrm{P}<0.05)$.Conclusion AQP3 expression in diarrhea rats colon were decreased, and the heavier the degree of diarrhea, the lower expression quantity.
\end{abstract}

AQPs (aquaporins) is a small molecule protein found in the cell membrane. It is widely expressed in plants and animals. In this study, through the establishment of SD rat model of different degrees of diarrhea, the expression of colonic mucosal epithelial cells AQP3, to explore the different degree of diarrhea, changes of colonic epithelial cells and the expression of AQP3, to further clarify the occurrence of diarrhea and development at the molecular level, so as to provide laboratory basis for the development of water channel protein agonists or the inhibitor, eventually provide a new way for the clinical treatment of diarrhea.

\section{Materials and Methods}

Materials. 40 healthy SD rats were divided into 4 groups: control group, mild diarrhea group, moderate diarrhea group and severe diarrhea group, 10 rats each with 180 to $200 \mathrm{~g}$ weight. The rats were randomly divided into two groups: control group, mild diarrhea group, moderate diarrhea group and severe diarrhea group. Senna decoction preparation: Senna add boiling water amount of 5min, two layers of gauze filtration, the filtrate was $5000 \mathrm{r} / \mathrm{min}$ centrifugation after $15 \mathrm{~min}$ decompressed and condensed into the content of crude drug was $1 \mathrm{~g} / \mathrm{mL}$ liquid, placed in the temperature of 4 DEG refrigerator spare. AQP3 antibodies (US Santa Cruz, Rabbit anti rat), two step immunohistochemical detection reagent (Beijing Chinese fir bridge) and PCR Kit (Shanghai, Roche Pharmaceutical Ltd). Electronic analytical balance METTLER-AB304-S (Shanghai Mettler Tor Instrument Co., Ltd.)

Methods. After the rats were fed with $7 \mathrm{~d}$, the experiment was carried out in single animal cage and kept in the animal room at constant temperature (25 C). The rats were randomly divided into control group, mild diarrhea group, moderate group and severe diarrhea group 4 groups, 10 rats in each group, 
respectively with saline, $0.3 \mathrm{~g} / \mathrm{ml}, 0.4 \mathrm{~g} / \mathrm{ml}, 0.5 \mathrm{~g} / \mathrm{ml} 2 \mathrm{ml}$ Senna Decoction orally, 1 day 1 times, continuous 7d model of diarrhea caused by different degree. After eighth days, the filter paper in the rat cage was used to continue the operation. The fecal mark of the rats was observed and the number of diarrhea was recorded. The expression of AQP3mRNA in colon tissue of colon specimen's detection in Liquid Nitrogen Precooling mortar after grinding according to the conventional method of extracting total RNA. RT-PCR method was used to determination of rat colon tissue AQP3 gene mRNA relative expression analysis. In mean + SD on body weight of rats, RT-PCR staining index and the 2- Delta Ct value, accord with normal distribution for single factor analysis of variance (Oneway-ANOVA), LSD was used for comparing with $\mathrm{P}<0.0522$, the difference was statistically significant.

\section{Results}

General Comparison of the Groups. Compared with the control group, the animals in the diarrhea group all showed weight loss $(\mathrm{P}<0.05)$, dry hair color, malaise, emaciation, curled up, tired, unresponsive, sluggish, and soft stools. The more severe the diarrhea is, the more obvious the performance is.

Comparison of Feces Water Content. The fecal water content in each diarrhea group (light/ moderate/ severe) was significantly higher than that in the control group (29.83 \pm 2.57$) \%$, the difference was statistically significant $(\mathrm{P}<0.01)$. The fecal water content of each diarrhea group was 22 , and the difference was statistically significant $(\mathrm{P}<0.05)$, indicating that the establishment of different levels of diarrhea model was successful.

Histopathology Analysis. Compared with the control group, the diarrhea group sticky secretion increased significantly, which was damaged after shedding of mucosa cells infiltrating the submucosa showed a large number of congested capillaries, glandular mucous layer swelling. The more severe the diarrhea is, the more obvious the change is.

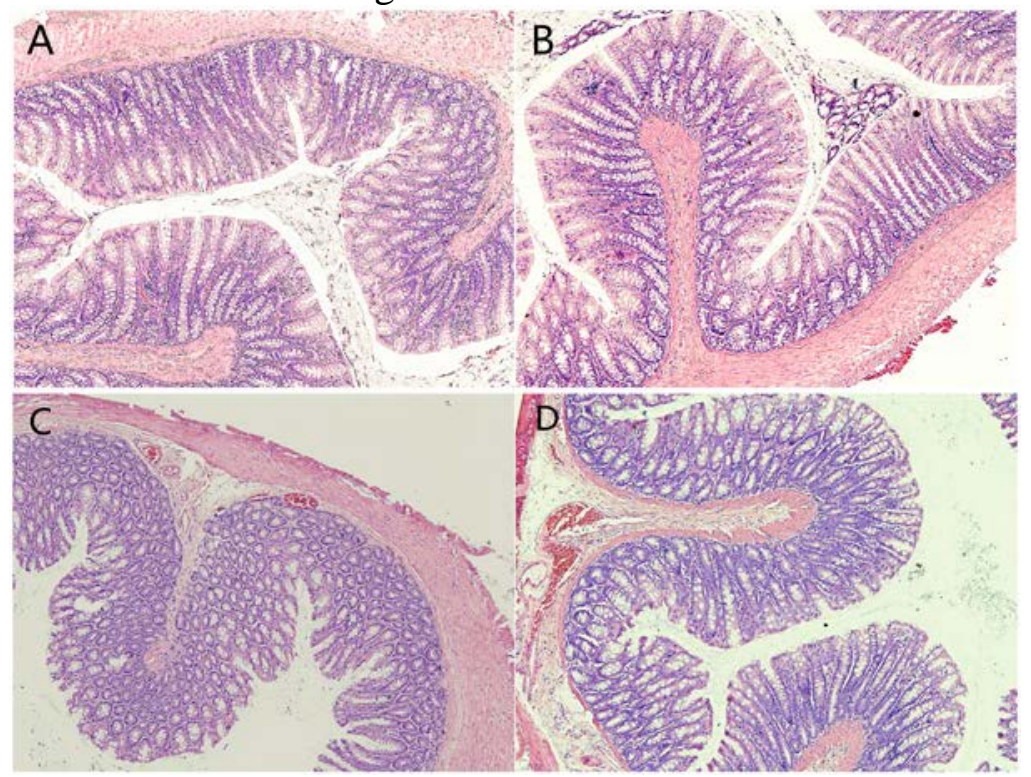

A. Control group B. Light diarrhea group C. Moderate diarrhea group D. Serve diarrhea group

Figure 1. Histopathology features of control group and diarrhea groups

Aquaporin 3 Expression in Colonic Tissues of Rats by Immunofluorescence Laser Confocal Microscop. The AQP3 positive cells were mainly expressed in the colonic mucosa, as shown in figure 2. The diarrhea group (mild / moderate / severe) staining index $(0.32+0.09 / 0.21+0.07 / 0.14+$ $0.05)$ was significantly lower than the control group $(0.59+0.11)$, the difference was statistically significant $(\mathrm{P}<0.01)$, indicating the colon decreased expression of AQP3 in diarrhea rats. The staining index of each diarrhea group was 22 , the difference was statistically significant $(P<0.05)$, indicating that the more severe the degree of diarrhea and diarrhea, the lower the expression of AQP3 protein. 


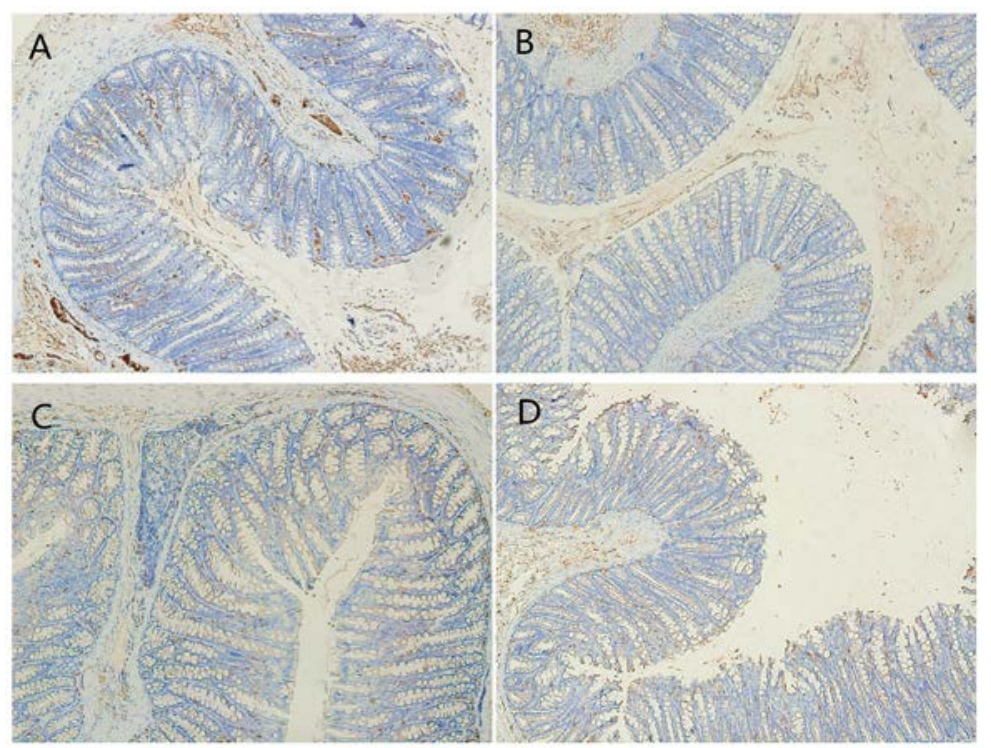

A. Control group B. Light diarrhea group C. Moderate diarrhea group D. Serve diarrhea group $(100 *)$

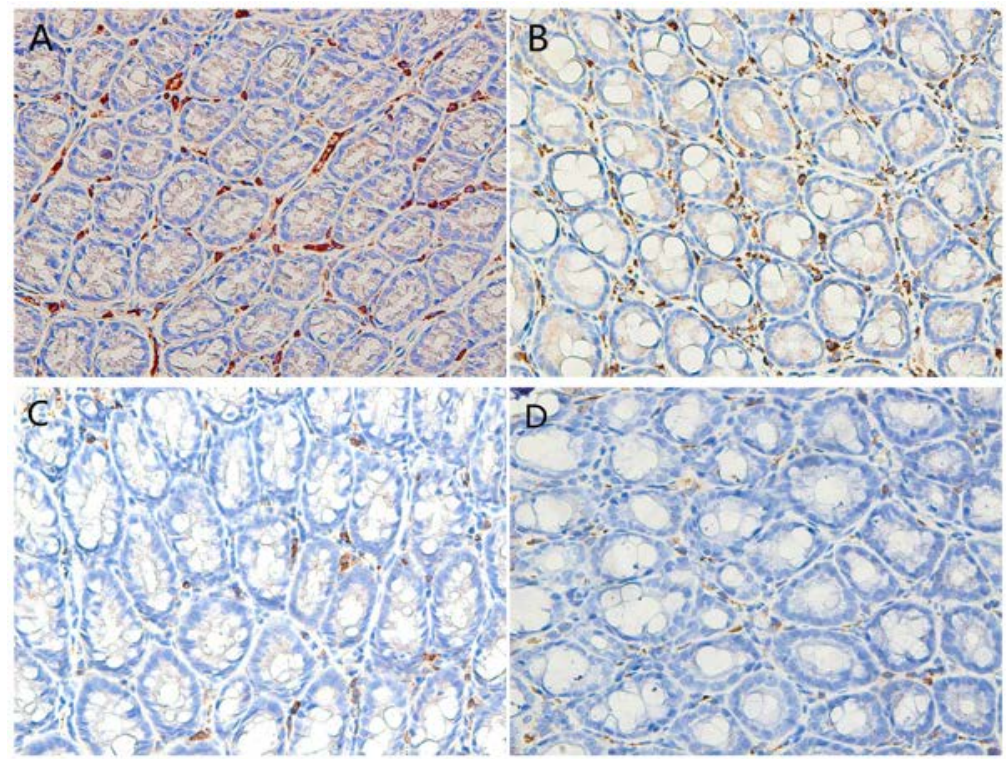

A. Control group B. Light diarrhea group C. Moderate diarrhea group D. Serve diarrhea group $(400 *)$

Figure 2. Aquaporin 3 expression of control group and diarrhea groups in Colonic Tissues of Rats by Immunofluorescence Laser Confocal Microscop

Aquaporin 3 Expression Level of Colon by Western Blot. In the control group and the expression of AQP3 was visible in the diarrhea group, diarrhea group (mild / moderate / severe) expression was significantly lower than the control group $(0.65 \pm 0.13) \%$, the difference was statistically significant $(\mathrm{P}<0.01)$, and the expression of 22 diarrhea group, the difference was statistically significant $(\mathrm{P}<0.01)$ further, the decrease of AQP3 protein expression in rat colonic diarrhea. With the aggravation of diarrhea degree, the expression of AQP3 protein decreased gradually.

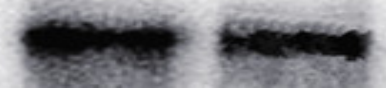

Control group, light diarrhea group, moderate diarrhea group, serve diarrhea group

Figure 3. Aquaporin 3 expression of control group and diarrhea groups by Western Blot

Expression Comparison of Rats' Colonic Tissues of Different Groups. RT-PCR results showed that: compared with the control group $(0.76+0.19)$, the diarrhea group (mild / moderate / severe) AQP3mRNA expression significantly decreased, the difference was statistically significant 
( $\mathrm{P}<0.01$ ), and the expression of 22 diarrhea group, the difference was statistically significant $(\mathrm{P}<0.05)$, indicating the colon in diarrhea rats the decreased expression of AQP3mRNA in. The more severe the diarrhea, the lower the expression of AQP3mRNA.

\section{Conclusions}

Diarrhea is a group of disease caused by many pathogenic factors and digestive diseases. It is a worldwide public health problem. In recent years, no progress has been made in the study and treatment of diarrhea at protein level and molecular level.

Aquaporin is expressed in many tissues and organs of the body, and play an important physiological role. They are closely related to the development of many diseases. Aquaporin 3 (AQP3), the major water channel protein in the digestive system, belongs to water glycerol channel proteins that transport water, glycerol, and small molecules. It is widely distributed in the digestive tract, from upper digestive tract to oral epithelial gastric mucosa, intestinal mucosal epithelium from the lower digestive tract to the anus, especially the small and large intestine parts distribution, and these parts are the key parts of the water inside and outside the cell transport. A lot of research has now proved that colon function is closely related to diarrhea and constipation. However, the correlation between diarrhea and AQP3 is not clear.

The results of this study are consistent with our observations that low expression or dysfunction of AQP3 may be involved in the pathogenesis of diarrhea by reducing the transport of water from the intestinal lumen to the intestinal wall, causing malabsorption of the water. In this study, the expression of AQP3 in the colon of diarrhea rats was decreased at the protein and molecular levels, and the lower the degree of diarrhea, the lower the expression level. Confirmed the occurrence of AQP3 in the control of diarrhea, diarrhea and further clarify the occurrence and development, so as to provide laboratory basis for the treatment of diarrhea at the gene level, provide new ideas for clinical treatment of diarrhea, but for the control mechanism of AQP3 gene expression, we also need further research.

\section{References}

[1] Matsuzaki T,Tajika Y, Ablimit A,el at.Aquaporins in the digestive system. Med Electron Microsc.2004, 37(2):71-80.

[2] Itoh A,Tsujikawa T,Yasuoka T,Nakahara T,et al.Natriuretic peptides up-regulate aquaporin 3in a human colonic epithelial cell line.Int J Mol Med.2004, 14(4):621-6.

[3] Verkman AS, Yang B,Song Y,et al.Role of water channels in fluid transport studied by phenotype analysis of aquaporin knockout mice.Exp Physiol.2000, 233S-241S.

[4] Ikarashi N, Baba K, Ushiki T, et al. The laxative effect of bisacodyl is attributable to decreased aquaporin-3 expression in the colon induced by increased PGE2 secretion from macrophages[J]. Am J Physiol Gastrointest Liver Physiol, 2011, 301(5): G887-G895.

[5] Benga, G., Popescu, O., Borza, V., Pop, V.I., Muresan, A., Mocsy, I., Brain, A. and Wrigglesworth, J.M. (1986) Water permeability in human erythrocytes: Identification of membrane proteins involved in water transport. European Journal of Cell Biology, 41, 252-262.

[6] Day, R.E., Kitchen, P., Owen, D.S., Bland, C., Marshall, L., Conner, A.C., Bill, R.M. and Conner, M.T. (2014) Human aquaporins: Regulators of transcellular water flow. Biochimica et Biophysica Acta, 1840, 1492-1506.

[7] Rojek, A., Praetorius, J., Frokiaer, J., Nielsen, S. and Fenton, R.A. (2008) A current view of the mammalian aquaglyceroporins. Annual Review of Physiology, 70, 301-327.

[8] Tradtrantip L, Tajima M, Li L, Verkman AS. Aquaporin water channels in transepithelial fluid transport. J Med Invest 2009; 56 Suppl: 179-184. 
[9] Laforenza U. Water channel proteins in the gastrointestinal tract. Mol Aspects Med, 2012, 33: 642-650.

[10] King LS, Yasui M. Aquaporins and disease: lessons from mice to humans. Trends Endocrinol Metab 2002; 13: 355-360.

[11] Ikarashi N, Kon R, Iizasa T, Suzuki N, Hiruma R,Suenaga K, Toda T, Ishii M, Hoshino M, Ochiai W, Sugiyama K. Inhibition of aquaporin-3 water channel in the colon induces diarrhea. Biol Pharm Bull 2012; 35:957-962.

[12] Ikarashi N, Ushiki T, Mochizuki T, Toda T, Kudo T, Baba K, Ishii M, Ito K, Ochiai W, Sugiyama K.Effects of magnesium sulphate administrationon aquaporin 3 in rat gastrointestinal tract. Biol Pharm Bull, 2011, 34: 238-242.

[13] Ikarashi N, Kon R, Iizasa T, et al. Inhibition of Aquaporin-3 Water Channel in the Colon Induces Diarrhea[J]. Biol Pharm Bull, 2012,35(6): 957-962. 